\title{
SHORT REPORT \\ Racial differences in seroprevalence of HAV and HEV in blood donors in the Western Cape, South Africa: a clue to the predominant HEV genotype?
}

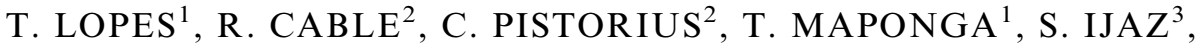 \\ W. PREISER ${ }^{1}$, R. TEDDER ${ }^{3,4}$ AND M. I. ANDERSSON ${ }^{1,5 *}$ \\ ${ }^{1}$ Division of Medical Virology, Department of Pathology, University of Stellenbosch, South Africa \\ ${ }^{2}$ Western Province Blood Transfusion Service, Cape Town, South Africa \\ ${ }^{3}$ Virus Reference Department, Public Health England, London, UK \\ ${ }^{4}$ NHS Blood and Transfusion, London, UK \\ ${ }^{5}$ Oxford University NHS Foundation Trust, Oxford, UK
}

Received 31 October 2016; Final revision 31 January 2017; Accepted 14 February 2017; first published online 30 March 2017

\section{SUMMARY}

Hepatitis E virus (HEV) infection is a major cause of acute hepatitis worldwide. This infection causes major water-borne outbreaks in low- and middle-income countries, whilst in industrialised countries this infection is zoonotic. These differences in epidemiology are related to different HEV genotypes. HEV genotype 3 is a zoonotic infection, whilst genotype 2 causes large outbreaks. This study determined the seroprevalence of HEV in blood donors from the Western Cape. Anti-hepatitis A virus (anti-HAV) antibody was detected in 184/300 (61\%) donors. Antibody to HEV (anti-HEV) was detected in 78 of 300 donors $(26 \%)$. It was highest in mixed race donors $(62 / 100)$, followed by white donors (23/100) and lowest in black donors $(19 / 100) P=$ 0.019 . Since it is thought that genotypes 1 and 2 predominate both viruses would be acquired by the oro-faecal route, it is surprising that HEV seroprevalence does not mirror that of HAV. We postulate that this may reflect differences in socio-economic status and consumption of dietary meat. So the marked divergence between HEV and HAV seroprevalence may be the result of different routes of transmission. Further data are needed to explore the risk factors associated with HEV infection.

Key words: Epidemiology, hepatitis E, liver infection, viral hepatitis.

Hepatitis E virus (HEV) is a common cause of acute viral hepatitis worldwide. While infections occur predominantly in low- and middle-income countries (LMIC), indigenous HEV infection also occurs in industrialised countries[1]. The epidemiological presentation of $\mathrm{HEV}$ infection differs substantially between these settings, linked to different HEV

\footnotetext{
* Author for correspondence: Dr M. Andersson, Division of Medical Virology, Department of Pathology, University of Stellenbosch, South Africa.

(Email: andersson_m@sun.ac.za)
}

genotypes. HEV genotype 1 and 2 are spread through exposure to faecally contaminated water in LMIC where sanitation is poor. During mass outbreaks pregnant women and young children are particularly vulnerable[2]. In contrast, HEV genotype 3 prevails in HIC (high-income countries) and is a zoonotic infection most likely acquired through the consumption of pig meat. It most commonly causes a subclinical acute infection in the immunocompetent with around only $1-2 \%$ of infections presenting with acute hepatitis, but may cause a severe chronic hepatitis in the immunocompromised host[1]. HEV genotype 4 is 


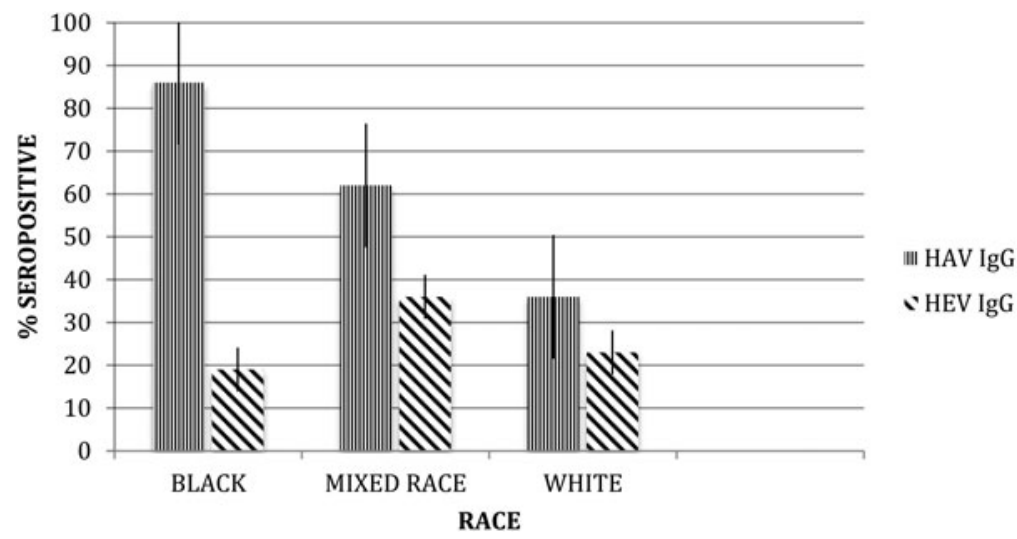

Fig. 1. Hepatitis A and hepatitis E seroprevalence in black, mixed race and white blood donors (with s.E.). HAV, hepatitis A virus; HEV, hepatitis E virus.

found in Asia and is also zoonotic. HEV infections in South Africa (SA) are thought to be due to HEV genotype 1 or $2[1,3]$.

Hepatitis A virus (HAV) infection is endemic in SA [4]. Recent studies have shown HAV contamination of water sources in SA[5]. Transmission of HAV occurs early in life where sanitation is inadequate and overcrowding is common[6]. HAV immunisation is not part of the EPI (Expanded Programme on Immunization).

SA has undergone tremendous political change since the first democratic elections in 1994, but the population remains largely divided along racial and socio-economic lines. Blood donors are self-selected volunteers who undergo rigorous screening before donation and their demography provides natural grouping in which to compare infection prevalence.

The aim of our study was to determine the seroprevalence of HAV and HEV antibodies in blood donors giving at the WPBTS (Western Province Blood Transfusion Service). Ethical permission for the study was granted by the Stellenbosch University Health Research Ethics Committee. All donors are routinely screened for HCV, HBV and HIV by serology and genomic testing (Tigris, Novartis Diagnostics). Following negative screening, panels of 100 residual samples were each randomly selected from black, mixed race and white blood donors. The majority were regular donors $(77 \%, 231 / 300) ; 54 \%$ $(162 / 300)$ were male; the mean age was 33.4 (range 16-66) years. Mean age and sex were not significantly different across the three race groups. Samples were further tested for anti-HAV (IgG) (Architect i2000, Abbott Diagnostics) and for anti-HEV IgG and IgM (Fortress Diagnostics, Antrim, UK).
Anti-HAV was detected in 184 of $300(61 \%)$ donors. The HAV seroprevalence increased with age from $31 \%(23 / 74)$ in those 21 years and younger, 68\% $(111 / 163)$ in those $22-45$ years and $79 \%$ (50/63) in those older than 46 years. It was highest in black donors (86/ $100)$, followed by mixed race donors $(62 / 100)$ and white donors (36/100). Men were less likely to be seropositive (92/162, 57\%) than women (90/138, 65\%).

Anti-HEV IgG was detected in 76 of 300 donors (25.3\%). The HEV seroprevalence increased with age from $5 \%(4 / 74)$ in those 21 years and younger, 26\% $(43 / 163)$ in those $22-45$ years and $46 \%(29 / 6363)$ in those older than 46 years $(P<0 \cdot 001)$. It was highest in mixed race donors $(33 / 100)$ followed by white donors (23/100) and was lowest in black donors (20/ 100) $(P=0.019)$ (Fig. 1). Men were more likely to be seropositive $(47 / 162,29 \%)$ than women $(29 / 138$, $21 \%$ ), although this difference was not significant. No sample contained detectable HEV IgM.

Since it is thought that HEV genotypes 1 and 2 are predominate in SA and both HEV and HAV would be acquired through the oro-faecal route in sub-Saharan Africa, it is surprising that the HEV prevalence does not mirror that of HAV as has been described elsewhere[7-9]. Instead, HEV seroprevalence is highest in mixed race donors and lowest in black donors. We postulate that this may reflect differences in socioeconomic status and level of dietary meat consumption. So the observed marked divergence between HEV and HAV seroprevalences in the three race groups may be a result of different routes of transmission. These data raise the question of whether HEV infection in SA may be predominantly zoonotic in origin implicating a predominance of HEV genotype 3 . These data are supported by a recent study from 
SA[10] and we have previously described HEV genotype 3 infection in SA in an HIV-infected individual [11] and a renal transplant recipient[12]. Further studies are needed to explore the risk factors associated with HEV infection and to identify possible sources of what may turn out to be a zoonotic infection after all.

\section{ACKNOWLEDGEMENTS}

The authors acknowledge the Polio Research Foundation for their financial support.

\section{REFERENCES}

1. Kamar N, Bendall R, Legrand-Abravanel $\mathrm{F}$, et al. Hepatitis E. Lancet 2012; 379(9835): 2477-2488. doi: 10.1016/S0140-6736(11)61849-7.

2. Teshale EH, Howard CM, Grytdal SP, et al. Hepatitis E epidemic, Uganda. Emerging Infectious Diseases 2010; 16(1): 126-129. doi: 10.3201/eid1601.090764.

3. Kim J-H, Nelson KE, Panzner U, Kasture Y, Labrique AB, Wierzba TF. A systematic review of the epidemiology of hepatitis E virus in Africa. BMC Infectious Diseases 2014; 14: 308. doi: 10.1186/1471-2334-14-308.

4. Jacobsen KH, Wiersma ST. Hepatitis A virus seroprevalence by age and world region, 1990 and 2005. Vaccine 2010; 28(41): 6653-6657. doi: 10.1016/j. vaccine.2010.08.037.

5. Chigor VN, Sibanda T, Okoh AI. Assessment of the risks for human health of adenoviruses, hepatitis A virus, rotaviruses and enteroviruses in the buffalo river and three source water dams in the Eastern Cape. Food and Environmental Virology 2014; 6(2): 87-98.

6. Shapiro CN, Margolis HS. Worldwide epidemiology of hepatitis A virus infection. Journal of Hepatology 1993; 18 (Suppl 2): S11-S14 (http://www.ncbi.nlm.nih.gov/ pubmed/8182265). Accessed 24 June 2014.

7. Jain P, Prakash S, Gupta S, et al. Prevalence of hepatitis A virus, hepatitis $B$ virus, hepatitis $C$ virus, hepatitis $\mathrm{D}$ virus and hepatitis $\mathrm{E}$ virus as causes of acute viral hepatitis in North India: a hospital based study. Indian Journal of Medical Microbiology 2013; 31(3): 261-265.

8. Zuin M, Caserta C, Romano L, Mele A, et al. Seroepidemiology of HEV and HAV in two populations with different socioeconomic levels and hygienic/sanitary conditions. European Journal of Clinical Microbiology and Infectious Diseases 2017; 36(3): 479-485.

9. Wong KH, Lui YM, Ng PS, et al. Epidemiology of hepatitis $\mathrm{A}$ and hepatitis $\mathrm{E}$ infection and their determinants in adult Chinese community in Hong Kong. Journal of Medical Virology 2004; 72(4): 538-544.

10. Madden R, Wallace A, Sonderup M, et al. Hepatitis E virus: Western Cape South Africa. World Journal of Gastroenterology 2016; 22(44): 9853-9859.

11. Andersson MI, Preiser W, Maponga TG, et al. Immune reconstitution hepatitis $\mathrm{E}$ : a neglected complication of antiretroviral therapy in Africa? AIDS 2013; 27(3): 487-489.

12. Andersson MI, Stead PA, Maponga T, van der Plas H, Preiser W. Hepatitis E virus infection: an underdiagnosed infection in transplant patients in Southern Africa? Journal of Clinical Virology 2015; 70: 23-25. doi: 10.1016/j.jcv.2015.06.081. 